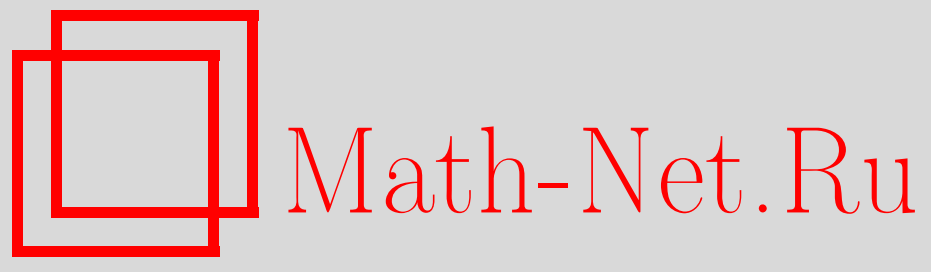

Р. Даншен, Аксиально-симметричные несжимаемые потоки с ограниченным вихрем, УМH, 2007, том 62, выпуск $3,73-94$

DOI: https://doi.org/10.4213/rm6761

Использование Общероссийского математического портала Math-Net.Ru подразумевает, что вы прочитали и согласны с пользовательским соглашением http://www . mathnet.ru/rus/agreement

Параметры загрузки:

IP : 54.210 .77 .194

26 апреля 2023 г., 14:16:48

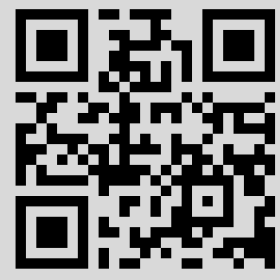




\section{Аксиально-симметричные несжимаемые потоки с ограниченным вихрем}

\section{Р. Даншен}

Эта статья посвящена доказательству результатов о глобальном существовании и единственности решений для трехмерных несжимаемых уравнений Эйлера со специальной геометрической структурой. Основное внимание уделяется так называемым аксиально-симметричным решениям без закручивания и геликоидальным решениям. Наша цель состоит в описании условий для вихря, максимально близких к условиям, сформулированным В. И. Юдовичем для двумерного случая.

Библиография: 20 названий.

\section{СОДЕРЖАНИЕ}

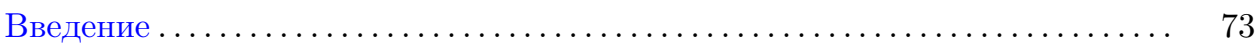

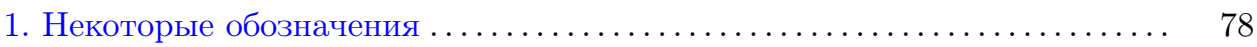

2. Априорные оценки для вихря и скорости ................... 80

3. Случай ограниченной области . . . . . . . . . . . . . . . . . . . . . 83

3.1. Доказательство единственности......................... 83

3.2. Доказательство существования ...................... 84

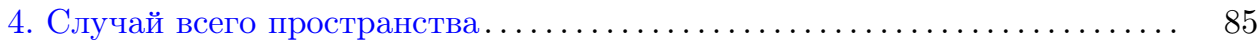

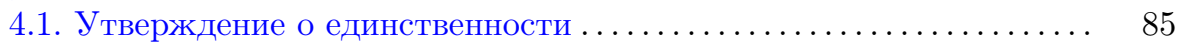

4.2. Доказательство существования ....................... 87

5. Геликоидальные решения . . . . . . . . . . . . . . . . . . . . . . . . . 90

5.1. Определения и свойства............................ 90

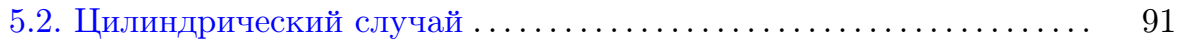

5.3. Случай всего пространства ........................ 91

6. Дальнейшие результаты и открытые проблемы ................ 92

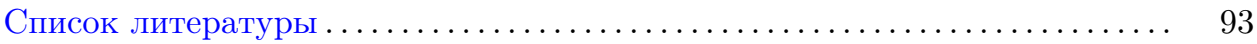

\section{Введение}

Настоящая работа посвящена доказательству результатов о глобальном существовании и единственности для несжимаемых уравнений Эйлера

$$
\left\{\begin{array}{l}
\partial_{t} v+v \cdot \nabla v+\nabla p=0 \\
\operatorname{div} v=0 \\
v_{\mid t=0}=v^{0}
\end{array}\right.
$$


в гладкой открытой области $\Omega$ пространства $\mathbb{R}^{3}$. Система (Е) снабжена граничными условиями скольжения вида $v \cdot n=0$ на $\partial \Omega$.

Математические исследования несжимаемых уравнений Эйлера имеют долгую историю. Локальное существование классических решений было впервые установлено Л. Лихтенштейном в серии статей, опубликованных в конце двадцатых годов (см., например, [1]). Существование двумерных глобальных классических решений было доказано В. Волибнером в 1933 г. (см. [2]). Коротко представим ключевые аргументы (в современной версии), которые приводят к глобальному существованию в случае $\Omega=\mathbb{R}^{2}$.

1. Законы сохранения вихря. В двумерном случае вихрь $\omega$ сводится к скалярной функции (а именно к функции $\omega:=\partial_{1} v^{2}-\partial_{2} v^{1}$ ) и удовлетворяет транспортному уравнению

$$
\partial_{t} \omega+v \cdot \nabla \omega=0
$$

В силу условия несжимаемости, поток $\psi$, построенный по $v$, сохраняет площадь. Следовательно, для любого $p \in[1,+\infty]$

$$
\|\omega(t)\|_{L^{p}}=\left\|\omega^{0}\right\|_{L^{p}} .
$$

2. Гёльдерова регулярность. Благодаря уравнению (1) гёльдеровы нормы вихря на любом интервале $\left[T_{1}, T_{2}\right]$ контролируются нормой функции $\nabla v$ в $L^{1}\left(T_{1}, T_{2} ; L^{\infty}\right)$. Точнее, для любого $\varepsilon \in(0,1)$ имеется некоторая константа $C$, зависящая только от $\varepsilon$, такая, что

$$
\sup _{t \in\left[T_{1}, T_{2}\right]}\|\omega(t)\|_{C^{\varepsilon}} \leqslant \min \left(\left\|\omega\left(T_{1}\right)\right\|_{C^{\varepsilon}},\left\|\omega\left(T_{2}\right)\right\|_{C^{\varepsilon}}\right) \exp \left(C \int_{T_{1}}^{T_{2}}\|\nabla v(t)\|_{L^{\infty}} d t\right) .
$$

3. Логарифмическая интерполяция. Используя тождество $\Delta v=\nabla^{\perp} \omega$ и тот факт, что $\operatorname{div} v=0$, имеем для любого $\varepsilon \in(0,1)$ неравенство

$$
\|\nabla v\|_{L^{\infty}} \leqslant C\|\omega\|_{L^{1} \cap L^{\infty}} \log \left(e+\frac{\|\omega\|_{C^{\varepsilon}}}{\|\omega\|_{L^{\infty}}}\right) .
$$

4. Глобальные априорные оценки. Подставляя (3) в (4) и применяя лемму Гронуолла, получаем (для всех $t \in \mathbb{R}$ ) оценку

$$
\|\nabla v(t)\|_{L^{\infty}} \leqslant C_{0} e^{C_{0}|t|}
$$

где $C_{0}$ можно выразить через нормы начальных данных.

Отметим, что подстановка этой последней оценки в (4) также дает глобальную (но очень плохую) оценку для $\|\omega(t)\|_{C^{\varepsilon}}$.

5. Единственность. Предполагая, что $v_{2}$ и $v_{1}$ оба удовлетворяют $(\mathrm{E})$, выводим с помощью основного энергетического метода следующее неравенство при Bcex $t \geqslant 0$ :

$$
\left\|\left(v_{2}-v_{1}\right)(t)\right\|_{L^{2}} \leqslant\left\|\left(v_{2}-v_{1}\right)(0)\right\|_{L^{2}} \exp \left(\int_{0}^{t}\left\|\nabla v_{1}(\tau)\right\|_{L^{\infty}} d \tau\right) .
$$

Следовательно, если оба решения $v_{2}$ и $v_{1}$ принадлежат $L^{2}$ и, кроме того, известно, что $\nabla v_{1} \in L^{1}\left(0, T ; L^{\infty}\right)$, то имеет место единственность решения на отрезке $[0, T]$. 
ЗАмечание 1. Отметим, что в отличие от существования единственность основывается на локальных аргументах. При этом размерность пространства не играет роли, а только предполагается, что система (Е) имеет решение $(v, p)$ с $\nabla v \in L^{1}\left(0, T ; L^{\infty}\right)$. Более того, $v$ не обязана принадлежать пространству $L^{2}$ (см., например, [3]).

В 1963 г. В. И. Юдович опубликовал в [4] новый результат о глобальной корректности несжимаемых уравнений Эйлера в гладкой ограниченной двумерной области. Предположения для начальных данных были более слабыми, чем в статье Волибнера: на самом деле, автор предполагал лишь, что начальный вихрь $\omega_{0}$ принадлежит $L^{\infty}$. Теперь, с одной стороны, глобальное существование можно получить с помощью сглаживания начальных данных. Но с другой стороны, доказательство единственности делается более сложным, чем в гладком случае, поскольку из ограниченности $\omega$ не следует ограниченность $\nabla v$. Значит, единственность теперь не выводится из неравенства (5).

Доказательство единственности у Юдовича опиралось на тот факт, что норма $\|\omega\|_{L^{p}}$ ограничена. Использованное им неравенство теперь стало классическим: существует константа $C$ такая, что

$$
\|\nabla v\|_{L^{p}} \leqslant \frac{C p^{2}}{p-1} \quad \text { для любого } \quad p \in(1, \infty) .
$$

Следовательно, выражение $\|\nabla v\|_{L^{p}} / p$ ограничено сверху константой, не зависящей от $p$, когда $p$ стремится к бесконечности. Правильное использование неравенства Гёльдера, а также обобщенной леммы Гронуолла (которую иногда называют леммой Осгуда) приводит к единственности.

Попутно заметим, что аргументация Юдовича годится и для случая всей плоскости, если вихрь имеет некоторое свойство убывания на бесконечности (см., например, [3]). В нашей тереме 2 мы увидим, что даже для $\omega$ и $v$ из $L^{\infty}$ можно получить единственность. Кроме того, недавно было обнаружено, что условие ограниченности $\|\omega\|_{L^{p}}$ не является необходимым для единственности. На самом деле, $\|\omega\|_{L^{p}}$ может (очень медленно) стремиться к бесконечности, когда $p$ стремится к бесконечности (см. [5] и [6]).

Для размерности $N=3$ вопрос о глобальной разрешимости системы (Е) остается нерешенным даже для малых начальных данных. Самое очевидное отличие от размерности два заключается в том, что вихрь $\omega$ является вектором и, следовательно, переносится потоком как векторное поле:

$$
\partial_{t} \omega+v \cdot \nabla \omega=\omega \cdot \nabla v
$$

Скорость можно вывести из вихря по закону Био-Савара, который для случая $\Omega=\mathbb{R}^{3}$ утверждает, что

$$
v(x)=\frac{1}{4 \pi} \int_{\mathbb{R}^{3}} \frac{(x-y) \times \omega(y)}{|x-y|^{3}} d y,
$$

а в более общем случае, если $\Omega$ достаточно гладкая, то

$$
\forall x \in \Omega \quad v(x)=-\int_{\Omega} \nabla_{x} K(x, y) \times \omega(y) d y,
$$


где $K$ - стандартная функция Грина, связанная с лапласианом задачи Дирихле в области $\Omega$.

Для общих трехмерных потоков не ясно, почему $\|\omega\|_{L^{\infty}}$ должно сохраняться при эволюции ${ }^{1}$, и двумерный подход к решению задачи (Е) не годится.

Однако при подходящих геометрических предположениях глобальное существование получается. Оно имеет место, когда

- данные являются трансляционно инвариантными (подобно двумерному случаю),

- данные геликоидальны (компоненты начального поля вихря постоянны на геликоидах, см. [8] и раздел 5),

- данные аксиально-симметричны без закручивания.

Для упрощения изложения остановимся на случае аксиально-симметричных данных без закручивания (понятие аксиально-симметричного векторного поля сейчас будет введено), а изучение геликоидальных решений отложим до раздела 5. Векторное поле $v$ называется аксиально-симметричным, если в цилиндрических координатах $(r, \theta, z)$

$$
v(r, \theta, z)=v_{r}(r, z) e_{r}+v_{z}(r, z) e_{z},
$$

где $e_{r}$ обозначает единичный (внешний) радиальный вектор и $e_{z}-$ единичный вертикальный вектор.

Простое вычисление показывает, что для этого случая вихрь $\omega$ сводится к

$$
\omega(r, \theta, z)=\omega_{\theta}(r, z) e_{\theta}, \quad \text { где } \quad \omega_{\theta}:=\partial_{z} v_{r}-\partial_{r} v_{z} \quad \text { и } \quad e_{\theta}=e_{z} \times e_{r} .
$$

Для аксиально-симметричных несжимаемых потоков величина $r^{-1} \omega_{\theta}$ играет ту же роль, что вихрь в двумерном случае. В самом деле, имеем

$$
\partial_{t}\left(r^{-1} \omega_{\theta}\right)+v \cdot \nabla\left(r^{-1} \omega_{\theta}\right)=0
$$

и, следовательно, $r^{-1} \omega_{\theta}$ имеет постоянную $L^{\infty}$-норму.

Этот факт использовался М.Р. Уховским и В.И. Юдовичем при доказательстве глобального существования аксиально-симметричных потоков в $\mathbb{R}^{3}$ с ограниченной энергией и начальным вихрем $\omega^{0} \in L^{2} \cap L^{\infty}$ таким, что $r^{-1} \omega^{0}$ принадлежит также $L^{2} \cap L^{\infty}$ (см. [9]). Напомним основные аргументы из этой работы.

1. Законы сохранения вихря. В силу (8), $L^{p}$-нормы $r^{-1} \omega$ сохраняются. Кроме того,

$$
\partial_{t} \omega_{\theta}+v \cdot \nabla \omega_{\theta}=r^{-1} \omega_{\theta} v_{r}
$$

и, следовательно, для любого $p \in[1,+\infty]$

$$
\|\omega(t)\|_{L^{p}} \leqslant\left\|\omega^{0}\right\|_{L^{p}}+\left\|r^{-1} \omega^{0}\right\|_{L^{p}} \int_{0}^{t}\|v(\tau)\|_{L^{\infty}} d \tau .
$$

2. Ограниченность $\|v\|_{L^{\infty}}$. Из закона Био-Савара получаем, что при любом $p>3$ (в [9] авторы берут $p=4)$ :

$$
\|v\|_{L^{\infty}} \lesssim\|v\|_{L^{2}}+\|\omega\|_{L^{p}},
$$

значит, используя тот факт, что $v$ имеет постоянную $L^{2}$-норму (это выполнено в силу энергетического тождества), подставляя (10) в (9) и применяя неравенство Гронуолла, получаем контроль над $\|v\|_{L^{\infty}}$ и $\|\omega\|_{L^{q}}$ при каждом $q \in[1,+\infty]$.

\footnotetext{
${ }^{1} \mathrm{~B}$ общем случае это не так: см. [7].
} 
3. Заключение. В частности, получаются экспоненциальные оценки для $\|\omega(t)\|_{L^{\infty}}$ (см. в [10] доказательство полиномиальных оценок). Теперь существование и единственность можно доказать так же, как в двумерном случае.

Однако, если говорить в терминах регулярности пространств Соболева, условие из [9] оказывается более сильным, чем условие, необходимое для локальной корректности в трехмерном случае. В самом деле, с одной стороны, общеизвестно, что имеется локальное существование в $H^{s}\left(\mathbb{R}^{3}\right)$ при $s>5 / 2$; с другой стороны, для того чтобы $r^{-1} \omega^{0}$ лежало в $L^{\infty}$ для любого $v^{0} \in H^{s}\left(\mathbb{R}^{3}\right)$, требуется $s>7 / 2$. Эта брешь была заполнена Т. Широтой и Т. Янагисавой в [11], где глобальное существование было доказано для любых аксиально-симметричных начальных векторных полей $v^{0}$ из $H^{s}\left(\mathbb{R}^{3}\right)$ при $s>5 / 2$.

Целью настоящей статьи является получение результата о глобальном существовании, который был бы максимально близок к двумерному случаю Юдовича: желательно иметь глобальный результат для начального аксиально-симметричного вихря в функциональном пространстве $E$, которое обладает той же масштабной инвариантностью, что и множество ограниченных функций, т.е. когда $E$ - множество ограниченных функций $f$ таких, что $r^{-1} f$ лежит в пространстве Лоренца $L^{3,1}\left(\mathbb{R}^{3}\right)$ (которое можно определить с помощью вещественной интерполяции по формуле $\left.L^{3,1}\left(\mathbb{R}^{3}\right)=\left(L^{\infty}\left(\mathbb{R}^{3}\right), L^{1}\left(\mathbb{R}^{3}\right)\right)_{\frac{1}{3}, 1}\right)$. Эта мотивация предваряет следующее утверждение.

ТЕОРема 1. Пусть $\Omega$ - или осесимметричная ограниченная область гладкости $C^{2, \alpha}$, или все пространство $\mathbb{R}^{3}$. Пусть начальный вихрь $\omega^{0}$ является аксиально-симметричной функцией в $L^{3,1}(\Omega) \cap L^{\infty}(\Omega)$ такой, что $r^{-1} \omega^{0} \in$ $L^{3,1}(\Omega)$. Обозначим через $v^{0}$ аксиально-симметричное соленоидальное векторное поле с вихрем $\omega^{0} e_{\theta}$, которое получается по закону Био-Савара (7). Тогда уравнения Эйлера (Е) имеют единственное глобальное решение $(v, \nabla p)$, причем $v \in \mathscr{C}_{w}\left(\mathbb{R} ; C_{\star}^{1}(\Omega)\right)$ u $\nabla p \in \mathscr{C}\left(\mathbb{R} ; C^{1-\varepsilon}(\Omega)\right)$ при любом $\varepsilon>0$. Кроме того,

$\omega \in L_{\mathrm{loc}}^{\infty}\left(\mathbb{R} ; L^{3,1}(\Omega) \cap L^{\infty}(\Omega)\right) \quad$ u $\quad\left\|r^{-1} \omega(t)\right\|_{L^{3,1}}=\left\|r^{-1} \omega^{0}\right\|_{L^{3,1}} \quad$ nри всех $\quad t \in \mathbb{R}$.

Выше через $C_{\star}^{1}(\Omega)$ обозначено пространство Зигмунда ограниченных непрерывных функций $f$ с областью определения $\Omega$ таких, что

$$
\|f\|_{C_{\star}^{1}}:=\|f\|_{L^{\infty}}+\sup _{(x, y) \in \widetilde{\Omega}^{2}} \frac{|f(x+y)+f(x-y)-2 f(x)|}{|y|^{2}}<\infty,
$$

где $\widetilde{\Omega}^{2}$ - множество таких пар $(x, y)$, что $x, x+y$ и $x-y$ принадлежат $\Omega$.

ЗАмечание 2. Конечно, в ограниченном случае $L^{3,1}(\Omega) \cap L^{\infty}(\Omega)=L^{\infty}(\Omega)$.

Формально доказательство теоремы 1 опирается на следующие три факта:

1) из (8) получаем, что $\left\|r^{-1} \omega(t)\right\|_{L^{3,1}}=\left\|r^{-1} \omega^{0}\right\|_{L^{3,1}}$ при всех $t \in \mathbb{R}$;

$2)$ в силу (7) найдется константа $C$, зависящая только от $\Omega$, такая, что $\left\|r^{-1} v_{r}\right\|_{L^{\infty}} \leqslant C\left\|r^{-1} \omega\right\|_{L^{3,1}}$

3) для осесимметричных потоков уравнение для $\omega_{\theta}$ сводится к

$$
\partial_{t} \omega_{\theta}+v \cdot \nabla \omega_{\theta}=r^{-1} v_{r} \omega_{\theta}
$$

ЗАмечАниЕ 3. Результаты о единственности приведены в утверждении 3 (случай ограниченной области $\Omega$ ) и в теореме $2\left(\right.$ случай $\Omega=\mathbb{R}^{2}$ ). 
Наша статья имеет следующую структуру. В разделе 1 даются определения нескольких функциональных пространств и вводится разложение ЛитлвудаПэли. Раздел 2 посвящен доказательству априорных оценок для векторных полей вихря и скорости. Теорема 1 для случая ограниченной области доказана в разделе 3 . В разделе 4 доказывается глобальное существование в случае $\Omega=\mathbb{R}^{3}$. Мы также получаем единственность, используя только $L^{\infty}$-норму вихря (или $C_{\star}^{1}$-норму скорости), не требуя условия убывания на бесконечности скорости или вихря (см. теорему 2). Этот результат, по нашему мнению, имеет самостоятельный интерес. Раздел 5 посвящен изучению геликоидальных решений с ограниченным вихрем. Некоторые дополнительные новые результаты и открытые проблемы перечислены в заключительной части статьи.

Автор благодарит А. Дютрифуа за полезные замечания о геликоидальных решениях.

\section{1. Некоторые обозначения}

Всюду в этой статье $L^{p}(\Omega)(1 \leqslant p \leqslant \infty)$ обозначает обычное пространство Лебега в области $\Omega$. Символ $\Omega$ будет опускаться, если смысл $\Omega$ ясен из контекста.

Дадим общее определение пространств Лоренца. В дальнейшем функция $f^{*}: \mathbb{R}^{+} \rightarrow \mathbb{R}^{+}$обозначает перестановку по убыванию функции $f$, которая задается формулой

$$
f^{*}(\tau)=\inf \left\{\lambda \in \mathbb{R}^{+}:|\{x \in \Omega:|f(x)|>\lambda\}| \leqslant \tau\right\} .
$$

ОПРЕДЕЛЕНИЕ 1 . Пусть $1 \leqslant p<\infty$ и $1 \leqslant q<\infty$. Обозначим через $L^{p, q}$ множество измеримых функций $f$, которые удовлетворяют условию

$$
\|f\|_{L^{p, q}}:=\frac{q}{p}\left(\int_{0}^{\infty}\left(\tau^{\frac{1}{p}} f^{*}(\tau)\right)^{q} \frac{d \tau}{\tau}\right)^{\frac{1}{q}}<\infty,
$$

а через $L^{p, \infty}-$ множество измеримых функций, для которых

$$
\|f\|_{L^{p, \infty}}:=\sup _{\tau>0} \tau^{\frac{1}{p}} f^{*}(\tau)<\infty .
$$

ЗАмЕчАНИЕ 4. Для полноты обозначений положим $L^{\infty, q}:=L^{\infty}$ при всех $q \in[1, \infty]$.

ЗАмечание 5. Отметим, что $L^{p, p}=L^{p}$. Кроме того, при $p \in(1, \infty)$ пространство $L^{p, q}$ совпадает с вещественным интерполяционным пространством $\left(L^{\infty}, L^{1}\right)_{\frac{1}{p}, q}$. Этот факт будет использоваться в настоящей статье многократно.

Читатель может обратиться к работе [12], где имеется подробная информация о пространствах Лоренца.

Теперь мы коротко опишем так называемое разложение Литлвуда-Пэли, которое будет использоваться при доказательстве теоремы 1 для случая $\Omega=\mathbb{R}^{3}$.

Пусть $\chi$ - некоторая гладкая радиальная функция $\chi$ с носителем, скажем, в шаре $B\left(0, \frac{4}{3}\right)$, равная 1 в некоторой окрестности шара $B\left(0, \frac{3}{4}\right)$ и такая, что 
функция $r \mapsto \chi\left(r e_{r}\right)$ не возрастает по $r$ вдоль $\mathbb{R}^{+}$. Обозначим $\varphi(\xi)=\chi(\xi / 2)-$ $\chi(\xi)$. Неоднородные бинарные блоки $\left(\Delta_{q}\right)_{q \geqslant-1}$ определяются по формулам:

$$
\Delta_{q}:=\varphi\left(2^{-q} D\right) \quad \text { при всех } \quad q \in \mathbb{N} \quad \text { и } \quad \Delta_{-1}=\chi(D) .
$$

Поскольку

$$
\sum_{q \geqslant-1} \varphi\left(2^{-q} \xi\right)=1 \quad \text { при всех } \xi \in \mathbb{R}^{N},
$$

имеем, что $\sum_{q \geqslant-1} \Delta_{q}=\operatorname{Id}$ в $\mathscr{S}^{\prime}\left(\mathbb{R}^{N}\right)$.

Мы также введем следующую функцию, срезающую низкие частоты:

$$
S_{q}:=\chi\left(2^{-q} D\right)=\sum_{p=-1}^{q-1} \Delta_{p} \quad \text { при любом } q \in \mathbb{N} .
$$

ЗАмЕчАНИЕ 6. Для любых распределений $u$ и $v$ из $\mathscr{S}^{\prime}$ носители спектра последовательности $\left(S_{q-1} u \Delta_{q} v\right)_{q \geqslant-1}$ лежат в бинарных ячейках. В самом деле, поскольку Supp $\chi \subset B\left(0, \frac{4}{3}\right)$, легко проверить, что

$$
\operatorname{Supp}\left(\mathscr{F}\left(S_{q-1} u \Delta_{q} v\right)\right) \subset\left\{\xi \in \mathbb{R}^{N}: \frac{1}{12} 2^{q} \leqslant|\xi| \leqslant \frac{10}{3} 2^{q}\right\} .
$$

Многие стандартные функциональные пространства можно характеризовать с помощью разложения Литлвуда-Пэли. В частности, это можно сделать для пространств Гёльдера $C^{s}$ при $s \in \mathbb{R}^{+} \backslash \mathbb{N}$ :

УтВеРЖДЕНИЕ 1. Пусть $s \in \mathbb{R}^{+} \backslash \mathbb{N}$. Пусть $u$ - распределение (обобщенная функция) умеренного роста. Тогда и принадлежит $C^{s}$, если и только если

$$
\sup _{q \geqslant-1} 2^{q s}\left\|\Delta_{q} u\right\|_{L^{\infty}}<\infty .
$$

Кроме того, выражение слева определяет норму в $C^{s}$, эквивалентную обычной норме.

ЗАмечАние 7. Отметим, что выражение (13) имеет смысл для любого вещественного $s$, даже для отрицательного. Однако в случае $s \in \mathbb{N}$ получаемое пространство шире, чем множество непрерывных ограниченных функций, имеющих непрерывные ограниченные производные всех порядков, не превосходящих $s$. Для избежания путаницы пространство, определенное в (13), обозначается $C_{\star}^{s}$ в случае $s \in \mathbb{N}$.

При $s=1$ можно показать, что пространство распределений $u$, удовлетворяющих (13), совпадает с пространством Зигмунда $C_{\star}^{1}$, определенным в (11).

При доказательстве существования потребуется сглаживание данных. Следующая лемма дает простой инструмент сглаживания в случае $\Omega=\mathbb{R}^{3}$.

ЛЕмма 1. Выполнены следующие неравенства: $п р и$ всех $n \in \mathbb{N}$

1) для любого $p \in[1,+\infty]$ имеем $\left\|S_{n} \omega\right\|_{L^{p}} \leqslant C\|\omega\|_{L^{p}}$;

2) для любого $p \in[1,+\infty]$ имеем $\left\|r^{-1} S_{n} \omega\right\|_{L^{p}} \leqslant C\left\|r^{-1} \omega\right\|_{L^{p}}$;

3) для любых $p \in(1,+\infty)$ u $q \in[1,+\infty]$ имеем $\left\|S_{n} \omega\right\|_{L^{p, q}} \leqslant C\|\omega\|_{L^{p, q}}$;

4) для любих $p \in(1,+\infty) u q \in[1,+\infty]$ имеем $\left\|r^{-1} S_{n} \omega\right\|_{L^{p, q}} \leqslant C\left\|r^{-1} \omega\right\|_{L^{p, q}}$. 
ДокАЗАТЕЛЬство. Первое неравенство является простым следствием определения $S_{n}$ и стандартных неравенств для свертки. Второе неравенство доказано в [10; лемма А.1].

Третье (соответственно четвертое) неравенство вытекает из первого (соответственно второго) в комбинации с вещественной интерполяцией.

\section{2. Априорные оценки для вихря и скорости}

В этом разделе предполагается, что функция Грина $K$, связанная с задачей Дирихле для $\Omega \subset \mathbb{R}^{3}$, имеет следующее свойство:

$$
\begin{gathered}
K \in \mathscr{C}^{2}\left(\Omega^{2}\right) \quad \text { и } \quad D_{X}^{k} K(X, Y)\left|\leqslant C_{k}\right| X-\left.Y\right|^{-1-k} \\
\text { для всех } \quad(X, Y) \in \Omega^{2} \quad \text { и } \quad k=0,1,2 .
\end{gathered}
$$

Отметим, что предположение $(\mathscr{H})$ выполнено в случае $\Omega=\mathbb{R}^{3}$, а также для ограниченных областей $\Omega$ с границей класса $C^{2, \alpha}$.

Следующая лемма является ключевой при доказательстве глобального существования в аксиально-симметричном случае.

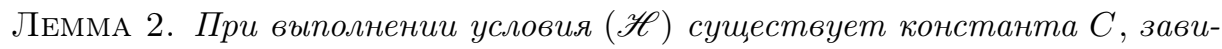
сящая только от $\Omega$, такая, что

$$
\left\|\frac{v_{r}}{r}\right\|_{L^{\infty}} \leqslant C\left\|\frac{\omega_{\theta}}{r}\right\|_{L^{3,1}} .
$$

ДокАзАТЕльство. Утверждение леммы вытекает из следующего неравенства, которое доказано в [11]:

$$
\forall X \in \Omega \quad\left|v_{r}(X)\right| \leqslant C \int_{\Omega} \min \left(1, \frac{r}{\left|X^{\prime}-X\right|}\right) \frac{\left|\omega_{\theta}\left(X^{\prime}\right)\right|}{\left|X-X^{\prime}\right|^{2}} d X^{\prime}
$$

для некоторой константы $C$, зависящей только от $\Omega$.

В самом деле, с одной стороны, если точка $X^{\prime} \in \Omega$ такова, что $\left|X^{\prime}-X\right| \leqslant r$, то $r^{\prime} \leqslant 2 r$. С другой стороны, если $\left|X^{\prime}-X\right|>r$, то $r^{\prime}\left|X-X^{\prime}\right|^{-1} \leqslant 2$. Следовательно, из (14) получаем, что

$$
\forall X \in \Omega \quad \frac{\left|v_{r}(X)\right|}{r} \leqslant 2 C \int \frac{1}{\left|X-X^{\prime}\right|^{2}} \frac{\left|\omega_{\theta}\left(X^{\prime}\right)\right|}{r^{\prime}} d X^{\prime} .
$$

Поскольку пространство $L^{\frac{3}{2}, \infty}$ является двойственным к $L^{3,1}$ (см. [12]) и отображение $Y \mapsto|Y|^{-2}$ принадлежит классу $L^{\frac{3}{2}}, \infty\left(\mathbb{R}^{3}\right)$, находим, что

$$
r^{-1}\left|v_{r}(X)\right| \leqslant C\left\|r^{-1} \omega_{\theta}\right\|_{L^{3,1}} \quad \text { при всех } \quad X \in \Omega,
$$

что дает нужный результат.

Для удобства читателя далее мы приводим доказательство неравенства (14).

Для $X=(x, y, z) \in \Omega$ обозначим $\check{X}:=(-x,-y, z)$. Доказательство основано на наблюдении, что имея осевую симметрию области $\Omega$, получаем для всех $\left(X, X^{\prime}\right) \in \Omega^{2}$ тождество

$$
K\left(\check{X}, X^{\prime}\right)=K\left(X, \check{X}^{\prime}\right)
$$


Для любого $z^{\prime} \in \mathbb{R}$ обозначим $I_{z^{\prime}}=\left\{r^{\prime} \in \mathbb{R}^{+}:\left(r^{\prime}, 0, z^{\prime}\right) \in \Omega\right\}$. Пусть $X=$ $(r \cos \theta, r \sin \theta, z) \in \Omega$. В силу инвариантности относительно поворота при вычислении $v_{r}(X)$ можно предполагать, что $\theta=0$. Используя аксиальную симметрию и закон Био-Савара (7), находим

$$
v_{r}(X)=\int_{-\infty}^{+\infty} \int_{I_{z^{\prime}}} \int_{-\pi}^{\pi} \partial_{z} K\left(X, X^{\prime}\right) \omega_{\theta}\left(X^{\prime}\right) \cos \theta^{\prime} r^{\prime} d \theta^{\prime} d r^{\prime} d z^{\prime},
$$

где $X^{\prime}=\left(r^{\prime} \cos \theta^{\prime}, r^{\prime} \sin \theta^{\prime}, z^{\prime}\right)$.

Следовательно, из (15) получаем

$$
v_{r}(X)=\int_{-\infty}^{+\infty} \int_{I_{z^{\prime}}} \int_{-\pi / 2}^{\pi / 2}\left(\partial_{z} K\left(X, X^{\prime}\right)-\partial_{z} K\left(\check{X}, X^{\prime}\right)\right) \omega_{\theta}\left(X^{\prime}\right) \cos \theta^{\prime} r^{\prime} d \theta^{\prime} d r^{\prime} d z^{\prime} .
$$

Чтобы доказать (14), достаточно проверить неравенство

$$
\begin{gathered}
\mid \underbrace{\int_{-\pi / 2}^{\pi / 2}\left(\partial_{z} K\left(X, X^{\prime}\right)-\partial_{z} K\left(\check{X}, X^{\prime}\right)\right) \omega_{\theta}\left(X^{\prime}\right) \cos \theta^{\prime} d \theta^{\prime} \mid}_{I} \\
\quad \leqslant C \int_{-\pi / 2}^{\pi / 2} \min \left(1, \frac{r}{\left|X^{\prime}-X\right|}\right) \frac{\left|\omega_{\theta}\left(X^{\prime}\right)\right|}{\left|X-X^{\prime}\right|^{2}} d \theta^{\prime} .
\end{gathered}
$$

Пусть $\theta^{*}$ обозначает единственное вещественное число $\theta^{\prime}$ из $[0, \pi / 2]$ такое, что $\left|X-X^{\prime}\right|=r$ (считается, что $\theta^{*}=0$, если $\left|X-X^{\prime}\right|>r$ при всех $\left.\theta^{\prime} \in[-\pi / 2, \pi / 2]\right)$. Разбиваем интеграл $I$ на три слагаемых: $I=I_{1}+I_{2}+I_{3}$, где

$$
I_{1}:=\int_{-\pi / 2}^{-\theta^{*}}(\cdots) d \theta^{\prime}, \quad I_{2}:=\int_{-\theta^{*}}^{\theta^{*}}(\cdots) d \theta^{\prime} \quad \text { и } \quad I_{3}:=\int_{\theta^{*}}^{\pi / 2}(\cdots) d \theta^{\prime} .
$$

В силу условия $(\mathscr{H})$, имеем

$$
\left|\partial_{z} K\left(X, X^{\prime}\right)\right| \leqslant C\left|X-X^{\prime}\right|^{-2} \quad \text { и }\left|\partial_{z} K\left(\check{X}, X^{\prime}\right)\right| \leqslant C\left|\check{X}-X^{\prime}\right|^{-2} .
$$

Раз $\left|\check{X}-X^{\prime}\right| \geqslant\left|X-X^{\prime}\right|$ при $\left|\theta^{\prime}\right| \leqslant \pi / 2$ и интервал $\left[-\theta^{*}, \theta^{*}\right]$ соответствует тем $\theta^{\prime}$, для которых $\left|X-X^{\prime}\right| \leqslant r$, мы заключаем, что $I_{2}$ удовлетворяет требуемому неравенству.

Теперь зафиксируем некоторый угол $\theta^{\prime} \in\left[\theta^{*}, \pi / 2\right]$ и обозначим $X_{t}:=(r \cos t$, $r \sin t, z)$ при $t \in[-\pi, 0]$. По формуле среднего имеем

$\partial_{z} K\left(X, X^{\prime}\right)-\partial_{z} K\left(\check{X}, X^{\prime}\right)=\pi r \int_{-\pi}^{0} D_{h} \partial_{z} K\left(X_{t}, X^{\prime}\right) \cdot e_{\theta} d t, \quad$ где $\quad D_{h}:=\left(\partial_{x_{1}}, \partial_{x_{2}}\right)$.

Используя $(\mathscr{H})$ и тот факт, что $\left|X^{\prime}-X_{t}\right| \geqslant\left|X^{\prime}-X\right|$ при всех $t \in[-\pi, 0]$, находим, что

$$
\left|\partial_{z} K\left(X, X^{\prime}\right)-\partial_{z} K\left(\check{X}, X^{\prime}\right)\right| \leqslant C r\left|X-X^{\prime}\right|^{-3}
$$

и тем самым

$$
\left|I_{3}\right| \leqslant r \int_{\theta^{*}}^{\pi / 2} \frac{\left|\omega_{\theta}\left(X^{\prime}\right)\right|}{\left|X-X^{\prime}\right|^{3}} d \theta^{\prime}
$$

Слагаемое $I_{1}$ можно оценить совершенно аналогично. Неравенство (14), а вместе с ним и лемма 2 доказаны. 
Следующая лемма, которая также доказывается с помощью закона БиоСавара с использованием двойственности между $L^{\frac{3}{2}, \infty}$ и $L^{3,1}$, будет весьма полезной.

Лемма 3. Существует константа $C$ такая, что

$$
\|v\|_{L^{\infty}} \leqslant C\|\omega\|_{L^{3,1}} .
$$

Установим теперь ряд априорных оценок в пространствах Лоренца для транспортного уравнения.

УТВЕРЖДЕНИЕ 2. Пусть $v$ - бездивергентное векторное поле с коэффициентами из $L^{1}\left(0, T ; C_{\star}^{1}\right)$ и такое, что $v \cdot n=0$ на $\partial \Omega$. Пусть а удовлетворяет уравнению $\partial_{t} a+\operatorname{div}(v a)=f$. Тогда при любъх $(p, q) \in[1,+\infty]^{2} u t \in[0, T]$ имеем

$$
\|a(t)\|_{L^{p, q}} \leqslant\|a(0)\|_{L^{p, q}}+\int_{0}^{t}\|f(\tau)\|_{L^{p, q}} d \tau .
$$

ДокАзАТЕльство. Пусть сначала $f \equiv 0$. Поскольку соленоидальное векторное поле $v$ принадлежит классу $L^{1}\left(0, T ; C_{\star}^{1}\right)$, ему соответствует поток $\psi$, сохраняющий меру Лебега (см., например, [3]). На самом деле, функция $\psi$ является (единственным) решением интегрального уравнения

$$
\forall t \in[0, T], \quad \forall x \in \Omega \quad \psi(t, x)=x+\int_{0}^{t} v(\tau, \psi(\tau, x)) d \tau .
$$

Теперь мы имеем $a(t, \psi(t, x))=a(0, x)$ при всех $t \in[0, T]$ и $x \in \Omega$. СледовательHO,

$$
\{y \in \Omega:|a(t, y)|>\lambda\}=\psi_{t}(\{x \in \Omega:|a(0, x)|>\lambda\}) .
$$

Раз $\psi$ сохраняет меру Лебега, оба множества в (17) имеют одинаковую меру, что, в силу определения 1 , влечет (16), если $f \equiv 0$.

Наконец, неравенство для произвольной силы $f \in L^{1}\left(0, T ; L^{p, q}\right)$ вытекает из формулы Дюамеля. Доказательство утверждения 2 завершено.

СледСтвиЕ 1. Пусть $v$ взято из утверждения 2 и нению вихря

$$
\partial_{t} \omega+\operatorname{div}(v \omega)=r^{-1} \omega v_{r}
$$

Тогда найдется константа $C$ такая, что при любъх $(p, q) \in[1,+\infty]^{2}$ и всех $t \in[0, T]$ справедливо неравенство

$$
\|\omega(t)\|_{L^{p, q}} \leqslant\left\|\omega^{0}\right\|_{L^{p, q}} \exp \left(C t\left\|r^{-1} \omega^{0}\right\|_{L^{3,1}}\right) .
$$

ДокАзАТЕЛЬство. Из утверждения 2 мы сразу получаем неравенство

$$
\|\omega(t)\|_{L^{p, q}} \leqslant\left\|\omega^{0}\right\|_{L^{p, q}}+\int_{0}^{t}\left\|r^{-1} v_{r} \omega\right\|_{L^{p, q}} d \tau .
$$

Теперь, используя определение пространств Лоренца, легко доказать, что

$$
\left\|r^{-1} v_{r} \omega\right\|_{L^{p, q}} \leqslant\left\|r^{-1} v_{r}\right\|_{L^{\infty}}\|\omega\|_{L^{p, q}} .
$$

Кроме того, в силу утверждения 2 и леммы 2 , имеем

$$
\begin{aligned}
\left\|r^{-1} v_{r}(t)\right\|_{L^{\infty}} & \leqslant C\left\|r^{-1} \omega(t)\right\|_{L^{3,1}}, \\
& \leqslant C\left\|r^{-1} \omega^{0}\right\|_{L^{3,1}} .
\end{aligned}
$$

Прямое применение леммы Гронуолла завершает доказательство следствия 1. 


\section{3. Случай ограниченной области}

Для упрощения обозначений мы сфокусируем наше внимание на случае эволюции при положительных временах. Чтобы получить аналогичные результаты для отрищательного времени, достаточно заменить $(v(t, x), p(t, x))$ на $(-v(-t, x), p(-t, x))$.

3.1. Доказательство единственности. Заметим, что в случае ограниченной области решение $v$ из теоремы 1 принадлежит пространству $\mathscr{C}\left(\mathbb{R}^{+} ; L^{2}\right)$. Поэтому единственность является простым следствием результата об устойчивости.

УТВЕРЖДЕНИЕ 3. Пусть область $\Omega$ принадлежит классу $C^{2, \alpha}$ и является ограниченной в $\mathbb{R}^{N}$. Пусть $\left(v^{1}, p^{1}\right)$ u $\left(v^{2}, p^{2}\right)$ - два решения системь (Е) на $[0, T] \times \Omega$ maкuе, что

$$
v^{1}, v^{2} \in L^{\infty}\left(0, T ; L^{\infty}\right) \cap \operatorname{Lip}\left([0, T] ; L^{2}\right), \quad \text { причем } \omega^{1} \in L^{\infty}\left(0, T ; L^{\infty}\right) .
$$

Тогда найдется константа $C$, зависящая только от $\Omega$ и от норм $\omega^{1}, v^{1}$ u $v^{2}$ в $L^{\infty}(0, T \times \Omega)$, такая, что если

$$
e^{-2}\left\|v^{2}(0)-v^{1}(0)\right\|_{L^{2}} \leqslant e^{-2 \exp (C T)},
$$

mо для всех $t \in[0, T]$

$$
e^{-2}\left\|v^{2}(t)-v^{1}(t)\right\|_{L^{2}} \leqslant\left\{e^{-2}\left\|v^{2}(0)-v^{1}(0)\right\|_{L^{2}}\right\}^{\exp (-C t)} .
$$

ДокАЗАТЕЛЬСтво. Мы используем рассуждениям Юдовича из [4]. Обозначив $\delta v:=v^{2}-v^{1}$ и $\delta v_{0}:=v^{2}(0)-v^{1}(0)$, имеем

$$
\left\{\begin{array}{l}
\partial_{t} \delta v+v^{2} \cdot \nabla \delta v=\nabla p^{1}-\nabla p^{2}-\delta v \cdot \nabla v^{1} \\
\delta v_{\mid t=0}=\delta v_{0}
\end{array}\right.
$$

Пусть $\varepsilon>0$ и $\delta_{\varepsilon}(t):=\sqrt{\varepsilon^{2}+\|\delta v(t)\|_{L^{2}}^{2}}$. Тогда очевидно, что

$$
\|\delta v(t)\|_{L^{2}} \leqslant \delta_{\varepsilon}(t) \quad \text { и } \quad \delta_{\varepsilon} \partial_{t} \delta_{\varepsilon}=\frac{1}{2} \frac{d}{d t} \delta_{\varepsilon}^{2}=-\int_{\Omega}\left(\delta v \cdot \nabla v^{1}\right) \cdot \delta v d x .
$$

Воспользовавшись неравенством Гёльдера и тем, что $\delta_{\varepsilon}>0$, находим, что

$$
\partial_{t} \delta_{\varepsilon} \leqslant \delta_{\varepsilon}^{1-\frac{1}{b}}\|\delta v\|_{L^{\infty}}^{\frac{1}{b}}\left\|\nabla v^{1}\right\|_{L^{b}} \quad \text { для любого } \quad b \in(1, \infty) .
$$

Напомним, что область $\Omega$ принадлежит классу $C^{2, \alpha}$ и является ограниченной. Тогда найдется некоторая константа $C$, зависящая только от $\Omega$, такая, что

$$
\|\nabla v\|_{L^{b}} \leqslant C \frac{b^{2}}{b-1}\|\omega\|_{L^{b}} \quad \text { для любого } \quad b \in(1, \infty) .
$$

Предположим, что $b$ является измеримой функцией времени $t$ (которая скоро будет задана), причем $b \geqslant 2$. Интегрируя по времени, получаем неравенство

$$
\delta_{\varepsilon}(t) \leqslant \delta_{\varepsilon}(0)+C \int_{0}^{t} b(\tau)\left\|\omega^{1}(\tau)\right\|_{L^{b(\tau)}}\left(\delta_{\varepsilon}(\tau)\right)^{1-\frac{1}{b(\tau)}}\|\delta v(\tau)\|_{L^{\infty}}^{1-\frac{1}{b(\tau)}} d \tau
$$


для некоторой константы $C$, не зависящей от $t \in[0, T]$. Следовательно, в силу того, что $\delta v$ и $\omega^{1}$ принадлежат $L^{\infty}\left(0, T ; L^{\infty}\right)$ и область $\Omega$ ограничена,

$$
\delta_{\varepsilon}(t) \leqslant \delta_{\varepsilon}(0)+C \int_{0}^{t} b(\tau)\left(\delta_{\varepsilon}(\tau)\right)^{1-\frac{1}{b(\tau)}} d \tau .
$$

Предполагая далее, что $\delta_{\varepsilon} \leqslant 1$ на $[0, T]$, выберем $b(t)=2-\log \delta_{\varepsilon}(t)$. Из предыдущего неравенства получаем (с точностью до смены константы $C$ ):

$$
\delta_{\varepsilon}(t) \leqslant \delta_{\varepsilon}(0)+C \int_{0}^{t} \delta_{\varepsilon}(\tau)\left(2-\log \delta_{\varepsilon}(\tau)\right) d \tau .
$$

Теперь из леммы Осгуда вытекает после некоторых выкладок, что

$$
e^{-2} \delta_{\varepsilon}(t) \leqslant\left(e^{-2} \delta_{\varepsilon}(0)\right)^{\exp (-C t)} \quad \text { всякий раз, когда } \quad \delta_{\varepsilon}(\tau) \leqslant 1 \quad \text { на }[0, t] .
$$

Устремляя $\varepsilon$ к нулю и используя соображения связности, можно завершить доказательство утверждения 3 .

\section{2. Доказательство существования.}

Первый шаг: регуляризация и равномерные оценки. Продолжим $\omega_{\theta}^{0}$ нулем вне $\Omega$ и обозначим через $\omega_{\theta}^{0, n}$ ограничение $S_{n} \omega_{\theta}^{0}$ на область $\Omega$. Определим теперь $v^{0, n}$ из $\omega_{\theta}^{0, n}$ по закону Био-Савара (7). Отметим, что в силу того, что срезающая спектральная функция в определении $S_{n}$ была радиальной, функция $\omega_{\theta}^{0, n}$ является аксиально-симметричной и тем же свойством обладает функция $v^{0, n}$.

Поскольку $\omega^{0, n}$ является гладкой, функция $v^{0, n}$ принадлежит $C^{r}(\Omega)$ для некоторого $r \in(1,2)$ (так как $\Omega$ принадлежит классу $C^{2, \alpha}$ и является ограниченной, это вытекает из теоремы 6.6 в [13]). Следовательно, уравнения Эйлера с начальными данными $v^{0, n}$ имеют локальное решение $\left(v^{n}, p^{n}\right)$, причем $v^{n} \in \mathscr{C}\left([0, T] ; C^{r}\right)$ (см., например, [14]). Давление можно найти с помощью [14; с. 188]. Теперь, воспользовавшись симметрией и следствием 1 , получим, что $\omega^{n} \in L^{\infty}\left(0, T ; L^{\infty}\right)$. В силу критерия Била-Като-Майды в ограниченной области (см. [14]), заключаем, что решение $\left(v^{n}, p^{n}\right)$ является глобальным и $v^{n} \in \mathscr{C}\left(\mathbb{R}^{+} ; C^{r}\right)$.

Второй шаг: сходимость в $L_{\mathrm{loc}}^{\infty}\left(\mathbb{R}^{+} ; L^{2}\right)$. Зафиксируем положительное $T$. При $n \geqslant m$ обозначим $\delta v_{m}^{n}:=v^{n}-v^{m}$. Последовательности $\left(v^{p}\right)_{p \in \mathbb{N}}$ и $\left(\omega^{p}\right)_{p \in \mathbb{N}}$ равномерно ограничены в $L^{\infty}(0, T \times \Omega)$. В силу утверждения 3 , найдется константа $C$, не зависящая от $n$ и $m$, такая, что если

$$
\left\|v^{0, n}-v^{0, m}\right\|_{L^{2}} \leqslant e^{2} e^{-2 \exp (C T)}
$$

то

$$
\forall t \in[0, T] \quad\left\|\delta v_{m}^{n}(t)\right\|_{L^{2}} \leqslant e^{2}\left(e^{-2}\left\|v^{0, n}-v^{0, m}\right\|_{L^{2}}\right)^{\exp (-C t)} .
$$

Заметим, что последовательность $\left(v^{0, p}\right)_{p \in \mathbb{N}}$ сходится в $L^{2}$. Следовательно, условие (21) выполнено при достаточно больших $n \geqslant m$, и $(22)$ означает, что $\left(v^{p}\right)_{p \in \mathbb{N}}$ является последовательностью Коши в $L^{\infty}\left(0, T ; L^{2}\right)$. Поэтому заключаем, что $\left(v^{p}\right)_{p \in \mathbb{N}}$ сходится к некоторому соленоидальному векторному полю $v$ в пространстве $L_{\mathrm{loc}}^{\infty}\left(\mathbb{R}^{+} ; L^{2}\right)$. 
Последний шаг: заключение. Интерполируя между полученным результатом о сходимости и равномерными оценками первого шага, заключаем, что $\left(v^{n}\right)_{n \in \mathbb{N}}$ стремится к $v$ в $\mathscr{C}\left(\mathbb{R}^{+} ; \bigcap_{\varepsilon>0} C^{1-\varepsilon}\right)$. Этого достаточно для перехода к пределу в каждом члене уравнения. Использование утверждения 2 и следствия 1 завершает доказательство.

ЗАмечАниЕ 8. Легко проверить, что построенное решение имеет постоянную энергетическую норму.

\section{4. Случай всего пространства}

В этом разделе доказывается теорема 1 для случая $\Omega=\mathbb{R}^{3}$.

4.1. Утверждение о единственности. В этом пункте основное внимание уделяется свойству единственности решений из теоремы 1 для случая $\Omega=\mathbb{R}^{3}$. Отметим, что из условий на начальные данные не понятно, почему поле вихря должно принадлежать пространству $L^{2}$. Таким образом, единственность не следует из доказанного выше утверждения 3.

В этом пункте будет доказана единственность для любой размерности $N \geqslant$ 2 при единственном предположении, что давление $p$ является ограниченной функцией времени со значениями в множестве $L_{\mathrm{L}}^{\infty}$, которое принадлежит пространству локально ограниченных по $x$ функций, имеющих не более чем логарифмический порядок роста на бесконечности, и что скорость $v$ принадлежит $\mathscr{C}\left([0, T] ; C_{\star}^{0}\right) \cap L^{1}\left(0, T ; C_{\star}^{1}\right)$ (см. определение пространства $C_{\star}^{0}$ в замечании 7$)$. Сравнивая это с утверждением Вишика о единственности из работы [6], отметим, что, с одной стороны, здесь не требуется убывания $v$ или $\omega$. А с другой стороны, вихрь в теореме Вишика не обязан принадлежать $L^{1}\left(0, T ; C_{\star}^{0}\right)$.

Наше доказательство основывается на (элементарном) парадифференциальном исчислении, введенном Ж.-М. Бони в [15]. Основная идея этой техники заключается в том, что произведение любых двух распределений умеренного роста $f$ и $g$ можно формально разложить в следующую сумму:

$$
f g=T_{f} g+T_{g} f+R(f, g)
$$

где оператор парапроизведения $T$ определен по формуле $T_{f} g:=\sum_{q} S_{q-1} f \Delta_{q} g$, а остаточный оператор $R$ равен $R(f, g):=\sum_{q} \Delta_{q} f\left(\Delta_{q-1} g+\Delta_{q} g+\Delta_{q+1} g\right)$.

Используя преобразования из книги [3] (см. главу 4), в предположении, что $p \in L^{\infty}\left(0, T ; L_{\mathrm{L}}^{\infty}\right)$, уравнения Эйлера можно переписать в виде системы, в которой имеется лишь одна неизвестная функция $v$ :

$$
\partial_{t} v+v \cdot \nabla v=\Pi(v, v)
$$

где билинейный оператор П определяется по формуле

$$
\Pi(v, w):=-\nabla(-\Delta)^{-1}\left(T_{\partial_{i} w^{j}} \partial_{j} v^{i}+T_{\partial_{j} v^{i}} \partial_{i} w^{j}\right)+\nabla T_{i, j} R\left(v^{i}, w^{j}\right) .
$$

Суммирование производится по повторяющимся индексам, а оператор $T_{i j}$ был определен в [3] (см. теорему 2.5.1).

Наш результат о единственности формулируется следующим образом. 
Теорема 2. Пусть $v^{1}$ и $v^{2}$ являются решениями модифицированных уравнений Эйлера $(\widetilde{\mathrm{E}})$ на отрезке $[0, T]$. Предполагается, ито v ${ }^{1}$ и v $v^{2}$ принадлежат пересечению пространств

$$
\mathscr{C}\left([0, T] ; C_{\star}^{0}\right) \cap L^{1}\left(0, T ; C_{\star}^{1}\right) .
$$

Если известно, что $v^{1}(0)=v^{2}(0)$, то $v^{1} \equiv v^{2}$ на множестве $[0, T] \times \mathbb{R}^{N}$.

ДоказАтельство. Разность $\delta v:=v^{2}-v^{1}$ удовлетворяет уравнению

$$
\partial_{t} \delta v+v^{2} \cdot \nabla \delta v=\Pi\left(\delta v, v^{1}\right)+\Pi\left(v^{2}, \delta v\right)-\delta v \cdot \nabla v^{1} .
$$

Утверждается, что билинейный оператор П удовлетворяет следующей оценке при любом $\varepsilon \in(0,1)$ :

$$
\forall q \geqslant-1 \quad\left\|\Delta_{q} \Pi(v, w)\right\|_{L^{\infty}} \lesssim(q+2) 2^{q \varepsilon} \min \left(\|v\|_{C^{-\varepsilon}}\|w\|_{C_{\star}^{1}},\|w\|_{C^{-\varepsilon}}\|v\|_{C_{\star}^{1}}\right) .
$$

Это легко выводится из (23). В самом деле, имеем (см. замечание 6 выше), что

$$
\Delta_{q}\left(T_{\partial_{i} w^{j}} \partial_{j} v^{i}\right)=\sum_{q^{\prime}=q-4}^{q+4} \Delta_{q}\left(S_{q^{\prime}-1} \partial_{i} w^{j} \Delta_{q^{\prime}} \partial_{j} v^{i}\right) .
$$

По этой причине и в силу того, что однородный оператор $\nabla(-\Delta)^{-1}$ имеет порядок -1 , а носители последовательности $\left(S_{q^{\prime}-1} \partial_{i} w^{j} \Delta_{q^{\prime}} \partial_{j} v^{i}\right)_{q^{\prime} \geqslant-1}$ принадлежат бинарным ячейкам, находим, что

$$
\begin{aligned}
\left\|\Delta_{q} \nabla(-\Delta)^{-1}\left(S_{q^{\prime}-1} \partial_{i} w^{j} \Delta_{q^{\prime}} \partial_{j} v^{i}\right)\right\|_{L^{\infty}} & \lesssim 2^{-q}\left\|S_{q^{\prime}-1} \nabla w\right\|_{L^{\infty}}\left\|\Delta_{q^{\prime}} \nabla v\right\|_{L^{\infty}}, \\
& \lesssim(q+2) 2^{q \varepsilon}\|\nabla w\|_{C_{\star}^{0}}\|v\|_{C^{-\varepsilon}} .
\end{aligned}
$$

Используя стандартный результат о непрерывности парапроизведения (см., например, [3]), мы также имеем

$$
\left\|\Delta_{q} \nabla(-\Delta)^{-1} T_{\partial_{i} w^{j}} \partial_{j} v^{i}\right\|_{L^{\infty}} \lesssim 2^{q \varepsilon}\|\nabla v\|_{C_{\star}^{0}}\|\nabla w\|_{C^{-\varepsilon-1}} .
$$

Поэтому первое слагаемое в правой части (23) удовлетворяет (25). Аналогичные неравенства можно доказать и для второго слагаемого в (23).

Изучим теперь остаточный член в (23). При $\varepsilon<1$ остаточный оператор $R$ отображает пространства $C^{-\varepsilon} \times C_{\star}^{1}$ и $C_{\star}^{1} \times C^{-\varepsilon}$ в $C^{1-\varepsilon}$. Поскольку $\nabla T_{i j}$ отображает $C^{1-\varepsilon}$ в $C^{-\varepsilon}$ (см. [3; теорема 2.5.1]), то выполнено неравенство (25).

Теперь заметим, что

$$
\left\|\Delta_{q}(v \cdot \nabla w)\right\|_{L^{\infty}} \lesssim(q+2) 2^{q \varepsilon}\|w\|_{C_{\star}^{1}}\|v\|_{C^{-\varepsilon}} .
$$

Это легко устанавливается с помощью разложения Бони:

$$
v \cdot \nabla w^{i}=T_{v^{j}} \partial_{j} w^{i}+\partial_{j}\left(v^{j}, w^{i}\right)+T_{\partial_{j} w^{i}} v^{j} .
$$

Продолжим доказательство единственности. Имея в своем распоряжении неравенства (25) и (26), уравнение (24) можно рассматривать как транспортное уравнение, связанное с векторным полем, с коэффициентами из $L^{1}\left(0, T ; C_{\star}^{1}\right)$ и с правой частью $\delta f$ такой, что для любого $\varepsilon \in(0,1)$

$$
\left\|\Delta_{q} \delta f\right\|_{L^{\infty}} \leqslant C(q+2) 2^{q \varepsilon}\left(\left\|v^{1}\right\|_{C_{\star}^{1}}+\left\|v^{2}\right\|_{C_{\star}^{1}}\right)\|\delta v\|_{C^{-\varepsilon}} .
$$


Оценки для такого транспортного уравнения были выписаны в [16] в лемме 2.5. Кратко объясним, как двигаться дальше. Во-первых, применяя оператор $\Delta_{q}$ к уравнению (24) и используя леммы о перестановках и $(27)$, получаем для всех $q \geqslant-1$ при $t \in[0, T]$

$$
\left\|\Delta_{q} \delta v(t)\right\|_{L^{\infty}} \leqslant \int_{0}^{t} C(q+2) 2^{(q+2) \varepsilon_{\tau}} V^{\prime}(\tau)\|\delta v\|_{C^{-\varepsilon_{\tau}}} d \tau
$$

где

$$
V(\tau):=\int_{0}^{\tau}\left(\left\|v^{1}\right\|_{C_{\star}^{1}}+\left\|v^{2}\right\|_{C_{\star}^{1}}\right) d \tau^{\prime}
$$

для любой непрерывной функции $\tau \mapsto \varepsilon_{\tau}$ со значениями в $\left[0, \frac{1}{2}\right]$.

Положим $\varepsilon_{t}:=C^{\prime} \int_{0}^{t} V^{\prime}(\tau) d \tau$ для некоторой константы $C^{\prime}$, которая будет выбрана позже. Умножая обе части неравенства $(28)$ на $2^{-(q+2) \varepsilon_{t}}$ и переходя к точной верхней грани по $q \geqslant-1$ и $\tau \in[0, t]$, приходим к неравенству

$$
\begin{aligned}
\sup _{q \geqslant-1} & \sup _{\tau \in[0, t]} 2^{-C^{\prime}(q+2) V(\tau)}\left\|\Delta_{q} \delta v(\tau)\right\|_{L^{\infty}} \\
& \leqslant \int_{0}^{t} C(q+2) 2^{C^{\prime}(q+2)(V(\tau)-V(t))} V^{\prime}(\tau)\|\delta v\|_{C^{-\varepsilon_{\tau}}} d \tau
\end{aligned}
$$

при условии, что $t$ лежит на отрезке $\left[0, T_{0}\right]$, где

$$
T_{0}=\sup \left\{t \in[0, T]: C^{\prime} \int_{0}^{t} V(\tau) d \tau \leqslant \frac{1}{2}\right\} .
$$

Следовательно,

$$
\sup _{q \geqslant-1} \sup _{\tau \in[0, t]} 2^{-C^{\prime}(q+2) V(\tau)}\left\|\Delta_{q} \delta v(\tau)\right\|_{L^{\infty}} \leqslant \frac{C}{C^{\prime} \log 2} \sup _{\tau \in[0, t]}\|\delta v\|_{C^{-\varepsilon_{\tau}}},
$$

и тем самым, если константа $C^{\prime}$ выбрана достаточно большой, то

$$
\sup _{\tau \in[0, t]}\|\delta v(\tau)\|_{C^{-\varepsilon_{\tau}}} \leqslant \frac{1}{2} \sup _{\tau \in[0, t]}\|\delta v(\tau)\|_{C^{-\varepsilon_{\tau}}} .
$$

Отсюда получается единственность на отрезке $\left[0, T_{0}\right]$. Теперь, поскольку $V$ принадлежит $L^{1}(0, T)$, можно повторить рассуждения конечное число раз и получить единственность на всем отрезке $[0, T]$. Теорема 2 доказана.

4.2. Доказательство существования. Доказательство будет проведено в четыре шага. Для простоты рассматривается эволюция при положительных временах. Аналогичные рассуждения с очевидностью дадут существование и при отрицательных временах.

Шаг 1. Построение глобальных гладких приближенных решений. Мы хо-

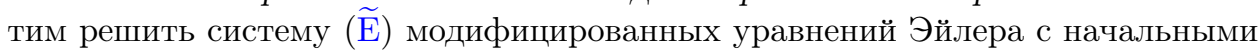
условиями $v^{0, n}:=S_{n} v^{0}$. Отметим, что поскольку поле $v^{0}$ является соленоидальным и имеет коэффициенты из $C_{\star}^{1}$, векторное поле $v^{0, n}$ также соленоидально и имеет коэффициенты, скажем, в пространстве $C^{\frac{3}{2}}$. Следовательно, 
в силу теоремы 4.2.1 из [3], найдется положительный момент времени $T^{*}$ (который мы выберем максимальным из возможных) такой, что ( $\widetilde{\mathrm{E}})$ с начальным условием $v^{0, n}$ имеет единственное решение $v^{n}$ в $\mathscr{C}\left(\left[0, T^{*}\right) ; C^{\frac{3}{2}}\right)$. Кроме того,

$$
T^{*} \int_{0}^{T^{*}}\left\|v^{n}(t)\right\|_{C_{\star}^{1}} d t=+\infty .
$$

Утверждается, что из условий для начальных данных следует, что $T^{*}=+\infty$. В самом деле, с одной стороны, отмечаем, что из аксиальной симметрии $v^{0}$ и из радиальности срезающей функции $\chi$ следует, что векторное поле $v^{0, n}$ также аксиально-симметрично. С другой стороны, в силу леммы 1 начальный вихрь $\omega^{0, n}$ удовлетворяет условиям

$$
\omega^{0, n} \in L^{3,1} \cap L^{\infty} \quad \text { и } \quad r^{-1} \omega^{0, n} \in L^{3,1} .
$$

Следовательно, воспользовавшись следствием 1, находим, что функция $\left\|\omega^{n}(t)\right\|_{L^{3,1} \cap L^{\infty}}$ остается ограниченной на любом ограниченном подмножестве интервала $\left[0, T^{*}\right)$. Теперь, объединяя закон Био-Савара, лемму 3 и разложение $v^{n}=S_{0} v^{n}+\left(\mathrm{Id}-S_{0}\right) v^{n}$, получаем, что

$$
\begin{aligned}
\left\|v^{n}\right\|_{C_{\star}^{1}} & \lesssim\left\|v^{n}\right\|_{L^{\infty}}+\left\|\nabla\left(v^{n}-S_{0} v^{n}\right)\right\|_{C_{\star}^{0}}, \\
& \lesssim\left\|\omega^{n}\right\|_{L^{3,1}}+\left\|\omega^{n}\right\|_{C_{\star}^{0}}, \\
& \lesssim\left\|\omega^{n}\right\|_{L^{3,1}}+\left\|\omega^{n}\right\|_{L^{\infty}} .
\end{aligned}
$$

Значит, последовательность $\left\|v^{n}(t)\right\|_{C_{\star}^{1}}$ остается ограниченной на любом ограниченном подмножестве из $\left[0, T^{*}\right)$. Отсюда заключаем, что $T^{*}=+\infty$.

Шаг 2. Равномерные оценки. Объединяя оценки из следствия 1 и лемм 1, 3, получаем, что при всех $t \in \mathbb{R}^{+}$

$$
\begin{aligned}
\left\|r^{-1} \omega^{n}(t)\right\|_{L^{3,1}} & \leqslant C\left\|r^{-1} \omega^{0}\right\|_{L^{3,1}}, \\
\left\|\omega^{n}(t)\right\|_{L^{3,1}} & \leqslant C e^{C t\left\|r^{-1} \omega^{0}\right\|_{L^{3,1}}\left\|\omega^{0}\right\|_{L^{3,1}}} \\
\left\|\omega^{n}(t)\right\|_{L^{\infty}} & \leqslant C e^{C t\left\|r^{-1} \omega^{0}\right\|_{L^{3,1}}}\left\|\omega^{0}\right\|_{L^{\infty}} .
\end{aligned}
$$

Учитывая соотношения $\left\|r^{-1} v^{n}\right\|_{L^{\infty}} \lesssim\left\|r^{-1} \omega^{n}\right\|_{L^{3,1}},\left\|v^{n}\right\|_{C_{\star}^{1}} \lesssim\left\|\omega^{n}\right\|_{L^{3,1}}+\left\|\omega^{n}\right\|_{L^{\infty}}$, заключаем, что

(a) последовательность $\left(v^{n}\right)_{n \in \mathbb{N}}$ ограничена в $\mathscr{C}\left(\mathbb{R}^{+} ; C_{\star}^{1}\right)$,

(b) последовательность $\left(\omega^{n}\right)_{n \in \mathbb{N}}$ ограничена в $L_{\mathrm{loc}}^{\infty}\left(\mathbb{R}^{+} ; L^{3,1} \cap L^{\infty}\right)$,

(c) последовательность $\left(r^{-1} \omega^{n}\right)_{n \in \mathbb{N}}$ ограничена в $L^{\infty}\left(\mathbb{R}^{+} ; L^{3,1}\right)$,

(d) последовательность $\left(r^{-1} v_{r}^{n}\right)_{n \in \mathbb{N}}$ ограничена в $L_{\text {loc }}^{\infty}\left(\mathbb{R}^{+} \times \mathbb{R}^{3}\right)$.

Шаг 3. Сходимость. Зафиксируем положительное T. Докажем, что $\left(v^{p}\right)_{p \in \mathbb{N}}$ является последовательностью Коши в $\mathscr{C}\left([0, T] ; C_{\star}^{0}\right)$.

Пусть $(m, n) \in \mathbb{N}^{2}$, причем $n \geqslant m$. Обозначим $\delta v_{m}^{n}:=v^{n}-v^{m}$. Тогда $\delta v_{m}^{n}$ удовлетворяет уравнению

$$
\partial_{t} \delta v_{m}^{n}+v^{n} \cdot \nabla \delta v_{m}^{n}=\underbrace{\Pi\left(v^{n}, \delta v_{m}^{n}\right)+\Pi\left(\delta v_{m}^{n}, v^{m}\right)-\delta v_{m}^{n} \cdot \nabla v^{m}}_{\delta f_{m}^{n}} .
$$


В предыдущем шаге установлено существование такого положительного числа $V_{T}$, что

$$
\forall p \in \mathbb{N} \quad\left\|v^{p}\right\|_{L^{\infty}\left(0, T ; C_{\star}^{1}\right)} \leqslant V_{T} .
$$

Кроме того, из неравенств $(25)$ и $(26)$ находим, что для любых $\alpha \in(0,1)$ и $q \geqslant-1$

$$
\left\|\Delta_{q} \delta f_{m}^{n}\right\|_{L^{\infty}} \lesssim(q+2) 2^{q \alpha}\left(\left\|v^{m}\right\|_{C_{\star}^{1}}+\left\|v^{n}\right\|_{C_{\star}^{1}}\right)\left\|\delta v_{m}^{n}\right\|_{C^{-\alpha}} .
$$

Рассуждая как при доказательстве теоремы 2, заключаем, что найдется некоторая константа $c$ (не зависящая от $T, n$ и $m$ ) такая, что

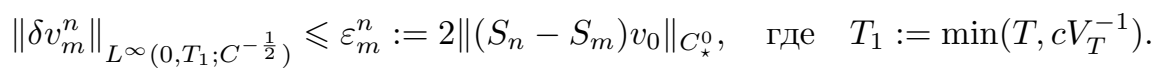

Применяя комплексную интерполяцию, находим, что

$$
\left\|\delta v_{m}^{n}\right\|_{L^{\infty}\left(0, T_{1} ; C_{\star}^{0}\right)} \leqslant\left\|\delta v_{m}^{n}\right\|_{L^{\infty}\left(0, T_{1} ; C^{-\frac{1}{2}}\right)}^{\frac{2}{3}}\left\|\delta v_{m}^{n}\right\|_{L^{\infty}\left(0, T_{1} ; C_{\star}^{1}\right)}^{\frac{1}{3}} \leqslant\left(\varepsilon_{m}^{n}\right)^{\frac{2}{3}}\left(2 V_{T}\right)^{\frac{1}{3}} .
$$

Аналогичные вычисления можно произвести для отрезков $\left[T_{1}, 2 T_{1}\right],\left[2 T_{1}, 3 T_{1}\right]$ и т.д. Получаем по индукции, что

$$
\left\|\delta v_{m}^{n}(t)\right\|_{C_{\star}^{0}} \leqslant 4 V_{T}\left(\varepsilon_{m}^{n}\right)^{\exp \left\{k \log \frac{2}{3}\right\}},
$$

когда $t \in\left[k T_{1}, \min \left(T,(k+1) T_{1}\right)\right]$. Следовательно, в силу определения $T_{1}$, имеем

$$
\left\|\delta v_{m}^{n}\right\|_{L^{\infty}\left(0, T_{1} ; C_{\star}^{0}\right)} \leqslant 4 V_{T}\left(\varepsilon_{m}^{n}\right)^{\exp \left\{\left(1+\frac{T V_{T}}{c}\right) \log \frac{2}{3}\right\} .}
$$

Значит,

$$
\varepsilon_{m}^{n} \leqslant C 2^{-n}\left\|v^{0}\right\|_{C_{*}^{1}},
$$

и тем самым последовательность $\left(v^{p}\right)_{p \in \mathbb{N}}$ сходится к некоторому соленоидальному векторному полю $v_{T} \in \mathscr{C}\left([0, T] ; C_{\star}^{0}\right)$.

Наконец, поскольку число $T$ было произвольным и имеются равномерные оценки в $L_{\mathrm{loc}}^{\infty}\left(\mathbb{R}^{+} ; C_{\star}^{1}\right)$, заключаем, что существует соленоидальное векторное поле $v$ в $L_{\mathrm{loc}}^{\infty}\left(\mathbb{R}^{+} ; C_{\star}^{1}\right) \cap \mathscr{C}\left(\mathbb{R}^{+} ; \bigcap_{\varepsilon>0} C^{1-\varepsilon}\right)$ такое, что $\left(v^{p}\right)_{p \in \mathbb{N}}$ стремится к $v$ в $L^{\infty}\left(\mathbb{R}^{+} ; \bigcap_{\varepsilon>0} C^{1-\varepsilon}\right)$. Кроме того, стандартное рассуждение из функционального анализа гарантирует, что $v \in \mathscr{C}_{w}\left(\mathbb{R}^{+} ; C_{\star}^{1}\right)$. В самом деле, пространство $C_{\star}^{1}$ является сопряженным к некоторому банахову пространству (а именно к пространству Бесова $\left.B_{1,1}^{-1}\right)$, которое содержит всюду плотное множество $\mathscr{C}_{c}^{\infty}$.

Шаг 4. Окончание доказательства. Доказанная сходимость в пространстве $L^{\infty}\left(\mathbb{R}^{+} ; \bigcap_{\varepsilon>0} C^{1-\varepsilon}\right)$ позволяет совершить предельный переход в уравнении $(\widetilde{\mathrm{E}})$. Далее, $\nabla p$ можно найти, используя теорему 2.5.1 и следствие 2.5 .1 из [3]. В итоге получается решение $(v, \nabla p) \in \mathscr{C}_{w}\left(\mathbb{R}^{+} ; C_{\star}^{1}\right) \times \mathscr{C}\left(\mathbb{R}^{+} ; \bigcap_{\varepsilon>0} C^{1-\varepsilon}\right)$ уравнений Эйлера (Е).

Кроме того, равномерные оценки для вихря, которые были установлены на втором шаге доказательства, дают включения $\omega \in L_{\mathrm{loc}}^{\infty}\left(\mathbb{R}^{+} ; L^{3,1} \cap L^{\infty}\right)$ и $r^{-1} \omega \in$ $L^{\infty}\left(\mathbb{R}^{+} ; L^{3,1}\right)$. Точнее, утверждение 2 влечет тождество

$$
\forall t \in \mathbb{R}^{+} \quad\left\|r^{-1} \omega(t)\right\|_{L^{3,1}}=\left\|r^{-1} \omega_{0}\right\|_{L^{3,1}} .
$$

Применяя теорему 2, получаем единственность. Это рассуждение завершает доказательство теоремы 1 для случая $\Omega=\mathbb{R}^{3}$. 


\section{5. Геликоидальные решения}

5.1. Определения и свойства. В работе [8] А. Дютрифуа ввел геликоидальные структуры, которые мы будем рассматривать в этом разделе. Для упрощения представления предполагается, что область $\Omega$ является или бесконечным круговым цилиндром радиуса $R$ с осью $(O z)$ (т.е. $\Omega=D(0, R) \times \mathbb{R}$ ), или всем пространством $\mathbb{R}^{3}$.

ОПРЕДЕЛЕНИЕ 2. Пусть $k$ - положительное число. Говорят, что соленоидальное векторное поле $v$ в $\Omega$ является геликоидальным, если

1) компоненты $v_{r}, v_{\theta}$ и $v_{z}$ поля $v$ постоянны на геликоидах $r=r_{0}, z=$ $z_{0}+k \theta$

2) в каждой точке $\Omega$ векторное поле $v$ ортогонально $h:=r e_{\theta}+k e_{z}$.

Отметим, что предельный случай $k=0$ соответствует аксиально-симметричному векторному полю без закручивания. Заметим также, что второе условие можно опустить (см. [17]). Эти более общие геликоидальные структуры совпадают в предельном случае $k=0$ с аксиально-симметричными структурами $c$ закручиванием.

Поскольку мы собираемся доказать глобальные результаты, с этого момента предполагаются выполненными два условия из приведенного выше определения. В работе [8] установлено, что если горизонтальное сечение $\Omega$ ограничено и начальное векторное поле $v^{0}$ является геликоидальным и достаточно гладким, то система (Е) имеет единственное глобальное решение $v$, которое также является геликоидальным.

Доказательство Дютрифуа основано на том факте, что вихрь $\omega$ удовлетворяет уравнениям

$$
k \omega=\omega_{z} h \quad \text { и } \quad\left(\partial_{t}+v \cdot \nabla\right) \omega_{z}=0,
$$

где $\omega_{z}$ обозначает вертикалъную компоненту вектора $\omega$.

Тогда, вводя поток $\psi$, отвечающий $v$, получаем (в очевидных обозначениях):

$$
\frac{\left|\omega\left(t, \psi_{t}(x)\right)\right|}{\sqrt{k^{2}+r^{2}\left(\psi_{t}(x)\right)}}=\frac{\left|\omega^{0}(x)\right|}{\sqrt{k^{2}+r^{2}}} .
$$

Поэтому в случае $\Omega=D(0, R) \times \mathbb{R}$ ясно, что $\omega$ остается ограниченным при всех временах, если $\omega^{0}$ был ограничен. Но тогда, применяя критерий Била-КатоМайды, получаем глобальное существование для достаточно гладких начальных условий.

В настоящем разделе мы задаемся вопросом о глобальной разрешимости геликоидальных уравнений Эйлера при выполнении единственного предположения об ограниченности вихря. Сформулируем основной результат.

Теорема 3. Пусть $\Omega=D(0, R) \times \mathbb{R}$ или $\mathbb{R}^{3}$. Предположим, что $v^{0}$ является соленоидальным и геликоидальным векторным полем в $\Omega$, имеющим ограниченный вихрь $\omega^{0}$ (в случае $\Omega=\mathbb{R}^{3}$ дополнительно предполагается, что $\left.\omega^{0} \in L^{2,1}\left(\mathbb{R}^{2} \times(-k \pi, k \pi)\right)\right)$. Тогда система $(\mathrm{E})$ имеет единственное глобальное геликоидальное решение с ограниченным вихрем.

Поскольку в случае всего пространства трудно контролировать низкие частоты, доказательства слегка различаются, в зависимости от того, является ли $\Omega$ цилиндром или $\mathbb{R}^{3}$. 
5.2. Цилиндрический случай. При доказательстве существования применяем подход Дютрифуа из [8], поскольку начальные данные были сглажены. Первый шаг требует особого внимания, так как геликоидальная структура должна сохраняться при сглаживании. Начальный вихрь $\omega^{0}$ имеет (в цилиндрических координатах) представление

$$
\omega^{0}(r, \theta, z)=F\left(r, \theta-k^{-1} z\right)\left(r e_{\theta}+k e_{z}\right)
$$

для некоторой функции $F$, зависящей только от двух переменных. Поэтому достаточно рассмотреть семейство функций $\left(F_{\varepsilon}\right)_{\varepsilon>0}$ класса $C^{1 / 2}(D(0, R))$, равномерно ограниченное в $L^{\infty}(D(0, R))$ и стремящееся к $F$ в $L^{\infty}$ *-слабо, и определить

$$
\omega_{\varepsilon}^{0}(r, \theta, z):=F_{\varepsilon}\left(r, \theta-k^{-1} z\right)\left(r e_{\theta}+k e_{z}\right) .
$$

Векторное поле $\omega_{\varepsilon}^{0}$ имеет свойства

$$
\omega_{\varepsilon}^{0} \in C^{1 / 2}\left(\mathbb{R}^{3}\right), \quad \operatorname{div} \omega_{\varepsilon}^{0}=0, \quad \omega_{\varepsilon}^{0} \cdot n=0 \quad \text { на } \quad \partial \Omega
$$

и является $2 k \pi$-периодическим по вертикальной переменной.

Тогда закон Био-Савара позволяет определить некоторое соленоидальное векторное поле $v_{\varepsilon}^{0}$ класса $C^{3 / 2}$, для которого $v_{\varepsilon}^{0} \cdot n=0$ на границе цилиндра. Заметим, что поле $v_{\varepsilon}^{0}$ является геликоидальным, но значение $v_{\varepsilon}^{0} \cdot h$ может не быть нулем. Однако $v_{\varepsilon}^{0} \cdot h$ является константой на $\Omega$.

Теперь на основании теоремы Дютрифуа из [8] (которая легко распространяется на случай, когда $v_{\varepsilon}^{0} \cdot h=\mathrm{const)}$ получаем глобальное геликоидальное решение $v_{\varepsilon}$ системы (Е) класса $C^{3 / 2}$ с начальными данными $v_{\varepsilon}^{0}$. При этом используется рассуждение, аналогичное тому, что применялось при обосновании аксиально-симметричного случая, когда делался предельный переход в (Е). В итоге получается геликоидальное решение $v$, отвечающее начальному условию $v^{0}$.

По правде сказать, единственность не вытекает из утверждения 3, поскольку область $\Omega$ не является ограниченной. Однако, используя периодичность скорости по вертикали и применяя стандартный энергетический метод, можно легко доказать (с обозначениями из утверждения 3), что функция

$$
\delta_{\varepsilon}(t):=\sqrt{\varepsilon^{2}+\|\delta v(t)\|_{L^{2}(D(0, R) \times(-k \pi, k \pi))}^{2}}
$$

удовлетворяет (19).

Начиная с этого места, единственность доказывается точно так же, как это делалось для утверждения 3.

5.3. Случай всего пространства. Единственность является простым следствием утверждения 3, поэтому более подробно рассмотрим часть теоремы 3 о существовании решения. Обозначим $\mathbb{R}_{k}^{3}:=\mathbb{R}^{2} \times(-k \pi, k \pi)$. Выполним некоторые формальные преобразования. Во-первых, используя закон Био-Савара и вертикальную периодичность вихря, получаем

$$
\forall X \in \mathbb{R}_{k}^{3} \quad|v(X)| \leqslant \frac{1}{4 \pi} \int_{-k \pi}^{k \pi} \int_{\mathbb{R}^{2}} \sum_{\ell \in \mathbb{Z}} \frac{\left|\omega\left(X^{\prime}\right)\right|}{\left|X_{h}-X_{h}^{\prime}\right|^{2}+\left|z-z^{\prime}-2 \ell k \pi\right|^{2}} d X_{h}^{\prime} d z^{\prime},
$$


где обозначено $X_{h}:=(x, y)$ и $X_{h}^{\prime}=\left(x^{\prime}, y^{\prime}\right)$. Следовательно,

$$
|v(X)| \leqslant C \int_{\mathbb{R}_{k}^{3}} \frac{\left|\omega\left(X^{\prime}\right)\right|}{\left|X_{h}-X_{h}^{\prime}\right|} d X^{\prime} .
$$

Тем самым доказано неравенство

$$
\|v\|_{L^{\infty}\left(\mathbb{R}^{3}\right)} \leqslant C\|\omega\|_{L^{2,1}\left(\mathbb{R}_{k}^{3}\right)} .
$$

Во-вторых, для геликоидальной скорости уравнение вихря сводится к

$$
k\left(\partial_{t}+v \cdot \nabla\right) \omega=\omega_{z}\left(v_{r} e_{\theta}-v_{\theta} e_{r}\right),
$$

поэтому, учитывая периодичность по вертикали и рассуждая как при доказательстве утверждения 2 , имеем для всех $t \in \mathbb{R}^{+}$

$$
\begin{aligned}
& k\|\omega(t)\|_{L^{2,1}\left(\mathbb{R}_{k}^{3}\right)} \leqslant k\left\|\omega^{0}\right\|_{L^{2,1}\left(\mathbb{R}_{k}^{3}\right)}+\int_{0}^{t}\|v\|_{L^{\infty}\left(\mathbb{R}^{3}\right)}\left\|\omega_{z}\right\|_{L^{2,1}\left(\mathbb{R}_{k}^{3}\right)} d \tau, \\
& k\|\omega(t)\|_{L^{\infty}\left(\mathbb{R}_{k}^{3}\right)} \leqslant k\left\|\omega^{0}\right\|_{L^{\infty}\left(\mathbb{R}_{k}^{3}\right)}+\int_{0}^{t}\|v\|_{L^{\infty}\left(\mathbb{R}^{3}\right)}\left\|\omega_{z}\right\|_{L^{\infty}\left(\mathbb{R}^{3}\right)} d \tau,
\end{aligned}
$$

т.е. поскольку $\omega_{z}$ переносится потоком и выполнено (32), находим, что

$$
\begin{aligned}
& k\|\omega(t)\|_{L^{2,1}\left(\mathbb{R}_{k}^{3}\right)} \leqslant k\left\|\omega^{0}\right\|_{L^{2,1}\left(\mathbb{R}_{k}^{3}\right)}+C\left\|\omega_{z}^{0}\right\|_{L^{2,1}\left(\mathbb{R}_{k}^{3}\right)} \int_{0}^{t}\|\omega\|_{L^{2,1}\left(\mathbb{R}_{k}^{3}\right)} d \tau, \\
& k\|\omega(t)\|_{L^{\infty}\left(\mathbb{R}_{k}^{3}\right)} \leqslant k\left\|\omega^{0}\right\|_{L^{\infty}\left(\mathbb{R}_{k}^{3}\right)}+C\left\|\omega_{z}^{0}\right\|_{L^{\infty}\left(\mathbb{R}^{3}\right)} \int_{0}^{t}\|\omega\|_{L^{2,1}\left(\mathbb{R}_{k}^{3}\right)} d \tau .
\end{aligned}
$$

Применяя лемму Гронуолла, заключаем, что

$$
\begin{aligned}
\|\omega(t)\|_{L^{2,1}\left(\mathbb{R}_{k}^{3}\right)} & \leqslant\left\|\omega^{0}\right\|_{L^{2,1}\left(\mathbb{R}_{k}^{3}\right)} e^{\frac{C}{k} t\left\|\omega_{z}^{0}\right\|_{L^{\infty}\left(\mathbb{R}^{3}\right)}}, \\
\|\omega(t)\|_{L^{\infty}\left(\mathbb{R}^{3}\right)} & \leqslant\left\|\omega^{0}\right\|_{L^{\infty}\left(\mathbb{R}^{3}\right)}+C^{-1}\left\|\omega_{z}^{0}\right\|_{L^{2,1}\left(\mathbb{R}_{k}^{3}\right)}\left(e^{\frac{C}{k} t\left\|\omega_{z}^{0}\right\|_{L^{\infty}\left(\mathbb{R}^{3}\right)}}-1\right) .
\end{aligned}
$$

Чтобы завершить доказательство теоремы 3, осталось лишь правильно сгладить данные. Это делается почти как в цилиндрическом случае (надо взять функции $F_{\varepsilon}$ с компактными носителями).

\section{6. Дальнейшие результаты и открытые проблемы}

1. Существование, доказанное в теореме 1, можно достаточно просто распространить на любую гладкую связную область с компактной границей. Что касается единственности, то она имеет место для решений с конечной энергией всякий раз, когда неравенство (20) выполнено в области $\Omega$.

2. Для упрощения изложения результатов и методов мы рассмотрели в данной статье лишь невязкую жидкость. В случае $\Omega=\mathbb{R}^{3}$ или $\Omega=\mathbb{R}^{2} \times \mathbb{S}^{1}$ большинство результатов, однако, распространяются и на вязкую жидкость. В частности, теорему 1 можно обобщить для аксиально-симметричных несжимаемых уравнений Навье-Стокса вида

$$
\left\{\begin{array}{l}
\partial_{t} v+v \cdot \nabla v-\nu \Delta v+\nabla p=0 \\
\operatorname{div} v=0
\end{array}\right.
$$


Сделав в точности те же предположения, что и в теореме 1, можно доказать результат о существовании с оценками, не зависящими от коэффициента вязкости $\nu$.

3. Имеется также возможность рассматривать неоднородные уравнения с внешней силой $f$, стоящей в правой части (Е). На самом деле, утверждение теоремы 1 остается верным, если предположить, что $f$ является аксиальносимметричной (без закручивания) и удовлетворяет условиям

$$
\operatorname{curl} f \in L_{\mathrm{loc}}^{1}\left(\mathbb{R} ; L^{\infty} \cap L^{3,1}\right) \quad \text { и } \quad r^{-1} \operatorname{curl} f \in L_{\mathrm{loc}}^{1}\left(\mathbb{R} ; L^{3,1}\right) .
$$

Доказательство оставляется читателю.

4. Теорема о глобальном существовании для аксиально-симметричных данных $v^{0}$ из $H^{s}$ при $s>5 / 2$, которая доказана в [11], теперь оказывается следствием теоремы 1 . На самом деле, утверждается, что если $v^{0} \in H^{s}$ - соленоидальное аксиально-симметричное векторное поле, то выполнены условия теоремы 1. Действительно, по теореме вложения Соболева находим, что $\omega^{0} \in L^{3,1} \cap L^{\infty}$ и если $s \in\left(\frac{5}{2}, \frac{7}{2}\right)$, то

$$
H^{s-2} \hookrightarrow L^{q} \quad \text { при } \quad 2 \leqslant q \leqslant q_{s}:=\frac{3}{\frac{7}{2}-s} .
$$

Следовательно, $D \omega^{0}$ (и тем самым $r^{-1} \omega^{0}$ ) принадлежит $L^{2} \cap L^{q_{s}}$. Так как $q_{s}>3$, по интерполяции получаем, что $r^{-1} \omega^{0} \in L^{3,1}$.

Объединяя наши глобальные оценки для $\|\omega\|_{L^{\infty}}$ с хорошо известным критерием Била-Като-Майды о продолжении (см. [18]), получаем глобальное существование в $H^{s}$.

5. Для аксиально-симметричных данных предположения теоремы 1 также выполнены, если известно, что $v^{0} \in B_{2,1}^{\frac{5}{2}}$, или (в более общем случае) если $v^{0} \in B_{p, 1}^{\frac{3}{p}+1}$ для некоторого $p \in[1,3)$. В самом деле, для достаточно малого $\varepsilon$ имеем

$$
B_{p, 1}^{\frac{3}{p}-1 \pm \varepsilon} \hookrightarrow L^{\frac{3}{1 \mp \varepsilon}},
$$

значит, совершая вещественную интерполяцию, получаем

$$
B_{p, 1}^{\frac{3}{p}-1}=\left(B_{p, 1}^{\frac{3}{p}-1-\varepsilon}, B_{p, 1}^{\frac{3}{p}-1+\varepsilon}\right)_{\left(\frac{1}{2}, 1\right)} \hookrightarrow\left(L^{\frac{3}{1+\varepsilon}}, L^{\frac{3}{1-\varepsilon}}\right)_{\left(\frac{1}{2}, 1\right)}=L^{3,1} .
$$

Следовательно, наша теорема 1 дает глобальное решение с вихрем из $L_{\mathrm{loc}}^{\infty}\left(\mathbb{R} ; L^{\infty}\right)$. Остается открытым вопрос: будет ли начальное условие регулярности класса $B_{p, 1}^{\frac{3}{p}+1}$ сохраняться все последующее время? Это, конечно, верно для малого временного интервала (см., например, [19]). Однако не ясно, можно ли применять критерий Била-Като-Майды в пространствах Бесова. Таким образом, даже при сохранении ограниченности вихря для всех временах не известно, сохраняется ли глобально критическая регулярность $B_{p, 1}^{\frac{3}{p}+1}$.

Отметим, что М. М. Вишик решил аналогичную проблему для двумерного случая. На самом деле, он смог обойти критерий Била-Като-Майды, остроумно использовав уравнения вихря. Его доказательство, однако, в решающей степени основано на том факте, что в случае двумерной несжимаемой жидкости в уравнении вихря отсутствует член растяжения (см. [20]). 
Р. ДАНШЕН

\section{Список литературы}

[1] L. Lichtenstein, "Über einige Existenzprobleme der Hydrodynamik", Math. Z., 32:1 (1930), 608-640.

[2] W. Wolibner, "Un theorème sur l'existence du mouvement plan d'un fluide parfait, homogène, incompressible, pendant un temps infiniment long", Math. Z., 37:1 (1933), 698-726.

[3] J.-Y. Chemin, "Fluides parfaits incompressibles", Astérisque, 230, 1995.

[4] В. И. Юдович, "Нестационарные течения в идеальной несжимаемой жидкости", Журн. вычисл. матем. и матем. физ., 3 (1963), 1032-1066.

[5] V.I. Yudovich, "Uniqueness theorem for the basic nonstationary problem in the dynamics of an ideal incompressible fluid", Math. Res. Lett., 2:1 (1995), 27-38.

[6] M. Vishik, "Incompressible flows of an ideal fluid with vorticity in borderline spaces of Besov type", Ann. Sci. École Norm. Sup. (4), 32:6 (1999), 769-812.

[7] D. Serre, "La croissance de la vorticité dans les écoulements parfaits incompressibles", C. R. Acad. Sci. Paris Sér. I Math., 328:6 (1999), 549-552.

[8] A. Dutrifoy, "Existence globale en temps de solutions hélicoïdales des équations d'Euler", C. R. Acad. Sci. Paris Sér. I Math., 329:7 (1999), 653-656.

[9] В. И. Юдович, М. Р. Уховский, "Осесимметричные течения идеальной и вязкой жидкости, заполняющей все пространство", ПММ, 32:1 (1968), 59-69; англ. пер.: M. R. Ukhovskii, V. I. Yudovich, "Axially symmetric flows of ideal and viscous fluids filling the whole space", J. Appl. Math. Mech., 32:1 (1968), 52-61.

[10] J. Ben Ameur, R. Danchin, "Limite non visqueuse pour les fluides incompressibles axisymétriques", Nonlinear partial differential equations and their applications. Collège de France seminar, vol. XIV (Paris, France, 1997-1998), Stud. Math. Appl., 31, North-Holland, Amsterdam, 2002, 29-55.

[11] T. Shirota, T. Yanagisawa, "Note on global existence for axially symmetric solutions of the Euler system", Proc. Japan Acad. Ser. A. Math. Sci., 70:10 (1994), 299-304.

[12] R. A. Hunt, "On $L(p, q)$ spaces", Enseignement Math. (2), 12 (1966), 249-276.

[13] D. Gilbarg, N.S. Trudinger, Elliptic partial differential equations of second order, Classics Math., Springer-Verlag, Berlin, 2001.

[14] A. Dutrifoy, "Precise regularity results for the Euler equations", J. Math. Anal. Appl., 282:1 (2003), 177-200.

[15] J.-M. Bony, "Calcul symbolique et propagation des singularités pour les équations aux dérivées partielles non linéaires", Ann. Sci. École Norm. Sup. (4), 14:2 (1981), 209-246.

[16] R. Danchin, "Évolution d'une singularité de type cusp dans une poche de tourbillon", Rev. Mat. Iberoamericana, 16:2 (2000), 281-329.

[17] A. Mahalov, E. S. Titi, S. Leibovich, "Invariant helical subspaces for the Navier-Stokes equations", Arch. Rational Mech. Anal., 112:3 (1990), 193-222.

[18] J. T. Beale, T. Kato, A. Majda, "Remarks on the breakdown of smooth solutions for the 3-D Euler equations", Comm. Math. Phys., 94:1 (1984), 61-66.

[19] Y. Zhou, "Local well-posedness for the incompressible Euler equations in the critical Besov spaces", Ann. Inst. Fourier (Grenoble), 54:3 (2004), 773-786.

[20] M. Vishik, "Hydrodynamics in Besov spaces", Arch. Ration. Mech. Anal., 145:3 (1998), 197-214. 